Babou André BATIONo ${ }^{1}$ Nieyidouba LAMIEN ${ }^{2}$

Nicole DeMERS ${ }^{3}$

Serigne KANDJI ${ }^{3}$

\title{
Culture du baobab Adansonia digitata L. (Bombacaceae) en planche maraîchère : une méthode pour simplifier sa récolte et favoriser sa propagation au Sahel
}

1 Institut de l'environnement et de recherches agricoles (Inera/Dpf) Département productions forestières 04 BP 8645, Ouagadougou 04 Burkina Faso

2 Institut de l'environnement et de recherches agricoles (Inera) Département productions forestières Station de recherche de Farâkoba BP 910, Bobo-Dioulasso

Burkina Faso

3 Coordination régionale pour l'Afrique de l'Ouest et du Centre du World Agroforestry Centre (Icraf-Wca) BP 320, Bamako Mali

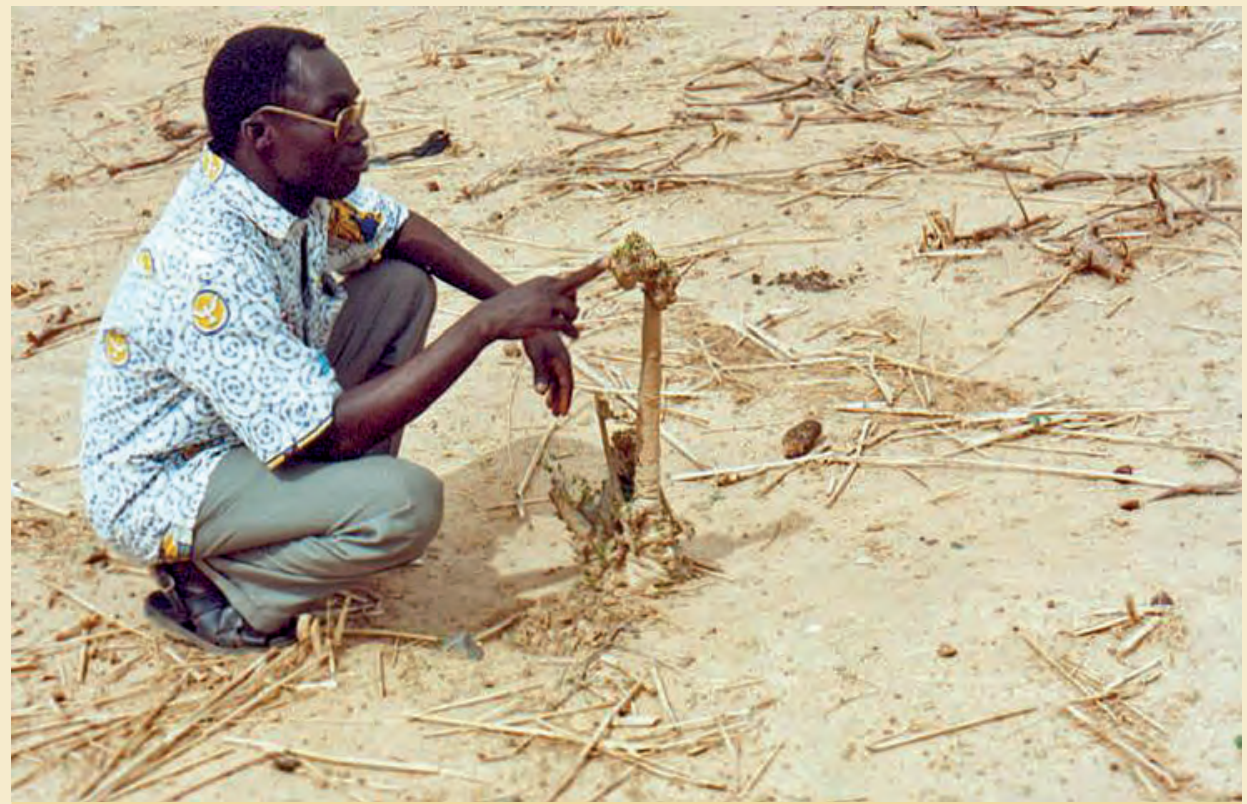

Photo 1.

Les multiples agressions entravent le développement des jeunes baobabs en milieu naturel. Ici un pied de baobab issu de la régénération naturelle âgé de 10-15 ans. Photo B. A. Bationo. 


\section{RÉSUMÉ}

\section{CULTURE DU BAOBAB ADANSONIA DIGITATA L. (BOMBACACEAE) EN PLANCHE MARAÎCHÈRE : UNE MÉTHODE POUR SIMPLIFIER SA RÉCOLTE ET FAVORISER SA PROPAGATION AU SAHEL}

Le baobab (Adansonia digitata L.) est l'une des principales espèces ligneuses alimentaires des pays sahéliens. Les feuilles et les fruits sont largement consommés. Sa régénération artificielle reste cependant marginale au Sahel. La technique de production maraîchère du baobab développée par le World Agroforestry Centre (Icraf) et les instituts nationaux de recherche agricole du Sahel apparaît comme une alternative pour accroître l'approvisionnement des populations en feuilles de baobab. L'étude entreprise en station et en milieu paysan a pour objectifs d'évaluer la productivité des planches de baobab soumises à différentes techniques de récolte, de suivre le comportement végétatif des plants après exploitation et de tester l'aptitude du baobab à la transplantation. Deux techniques de récolte sont appliquées : la récolte sur pied en épargnant les bourgeons terminaux et la récolte par coupes rases successives à $5 \mathrm{~cm}$ du sol. Les essais de transplantation sont réalisés avec trois stades (I, II, III) de développement. Les résultats montrent que le rendement en feuilles dépend de la saison et de la technique de récolte. Un an après la germination, les rendements sont en moyenne de $2 \mathrm{~kg} / \mathrm{m}^{2}$ et $0,6 \mathrm{~kg} / \mathrm{m}^{2}$ respectivement sur les planches soumises à la récolte sur pied et à la coupe rase. Les plants transplantés au stade III donnent les meilleurs taux de survie et de croissance. La technique de récolte des feuilles sur pied, en épargnant les bourgeons terminaux, permet ainsi d'intensifier la production de feuilles fraîches et de plants vigoureux pour régénérer les parcs agroforestiers.

Mots-clés : baobab, parc agroforestier, régénération, jardin.

B. A. BATIONO, N. LAMIEN, N. DemerS, S. KandJI

ABSTRACT

\section{CULTIVATION OF THE BAOBAB (ADANSONIA DIGITATA L. - BOMBACACEAE) AS A MARKET GARDEN CROP: A METHOD TO SIMPLIFY HARVESTING AND PROMOTE BAOBAB PROPAGATION IN THE SAHEL}

The baobab (Adansonia digitata L.) is one of the main tree species used for food in Sahelian countries. Its leaves and fruits are widely consumed. However, artificial regeneration of the baobab is marginal. Production of fresh baobab leaves in the market gardens run by the World Agroforestry Centre (ICRAF) and national agriculture research institutes in the Sahel may be an alternative way of increasing the supply of baobab leaves as a vegetable crop. This study, undertaken both on research stations and in crop fields, aimed to assess the productivity of baobab plots where different harvesting techniques are used, to monitor the vegetative behaviour of plants after cropping and to test the aptitude of baobabs for transplantation. Two techniques for harvesting leaves were applied: (1) harvesting from the mother tree without harming the terminal bud, (2) harvesting by successive cuttings $5 \mathrm{~cm}$ above the ground. Transplanting tests were carried out at three stages (I, II, III) of development. The results show that the leaf yield depends on the harvesting technique used and the season. One year after seed germination, leaf yields averaged $2 \mathrm{~kg} / \mathrm{m}^{2}$ when harvested from the mother-plant and $0.6 \mathrm{~kg} / \mathrm{m}^{2}$ when harvested by successive cuttings. The best transplantation results were obtained with seedlings at stage III. Harvesting from saplings without harming the terminal bud intensifies the production of fresh leaves and produces vigorous plant stock for agroforestry schemes.

Keywords: baobab, agroforestery, regeneration, market garden

\section{RESUMEN}

\section{CULTIVO DEL BAOBAB ADANSONIA \\ DIGITATA L. - BOMBACACEAE EN CAMA HORTÍCOLA: UN MÉTODO PARA SIMPLIFICAR SU COSECHA Y FAVORECER SU PROPAGACIÓN EN EL SAHEL}

El baobab (Adansonia digitata L.) es una de las principales especies leñosas alimenticias de los países del Sahel. Sus hojas y frutos son muy consumidos. No obstante, su regeneración artificial sigue siendo escasa en el Sahel. La técnica de producción hortícola del baobab, desarrollada por el World Agroforestry Centre (ICRAF) y los centros nacionales de investigaciones agrícolas del Sahel, se muestra como una alternativa para aumentar el suministro de hojas de baobab para las familias. El estudio, realizado en estación experimental y en medio campesino, tiene como objetivo evaluar la productividad de camas de baobab sometidas a diferentes técnicas de cosecha, observar el comportamiento vegetativo de las plantas tras su explotación y probar la aptitud al trasplante del baobab. Se emplean dos técnicas de cosecha: la cosecha en pie preservando las yemas terminales y la cosecha mediante cortas a hecho sucesivas a $5 \mathrm{~cm}$ del suelo. Los ensayos de trasplante se efectúan en tres fases de desarrollo (I, II, III). Los resultados ponen de manifiesto que el rendimiento en hojas depende de la temporada y de la técnica de cosecha. Un año después de la germinación, los rendimientos obtenidos muestran un promedio de $2 \mathrm{~kg} / \mathrm{m}^{2}$ y $0,6 \mathrm{~kg} / \mathrm{m}^{2}$, respectivamente, en las camas con cosecha en pie y en las de corta a hecho. Los plantones trasplantados en la fase III proporcionaron las mejores tasas de supervivencia y de crecimiento. Así pues, la técnica de cosecha de las hojas en pie preservando las yemas terminales permite intensificar la producción de hojas frescas y de plantones vigorosos para regenerar los parques agroforestales.

Palabras clave: baobab, parque agroforestal, regeneración, huerto 


\section{Introduction}

Le baobab (Adansonia digitata L.) est l'une des principales espèces ligneuses des parcs agroforestiers au Sahel. Les études de hiérarchisation des préférences paysannes entreprises depuis 1990 par le World Agroforestry Centre (Icraf) et les instituts nationaux de recherche agricole le placent parmi les cinq espèces prioritaires dans plusieurs régions du Mali, du Burkina Faso, du Niger et du Sénégal (Ouedraogo, 1995 ; CISSE, 1995). Les feuilles et la pulpe des fruits sont connues pour leur richesse en éléments nutritifs, notamment en vitamines A et C (LEAKEY, 1999). Ces produits font l'objet d'un commerce national et sous-régional (NIKIEMA, 1997) et procurent des revenus substantiels aux ménages sahéliens (BonKoungou et al., 1997), surtout aux femmes (LAMIEN, Traore, 2002).

Malgré cette importance économique, le baobab occupe une place marginale dans les campagnes de reboisement dans les pays du Sahel (CISSE, 1995 ; CES/AgF, 2000). Jusqu'à présent, les techniques classiques de régénération du baobab consistent à élever les plants en pépinière dans des pots en polyéthylène pendant 2 à 4 mois puis à les planter en milieu naturel au moment où leur hauteur est de l'ordre de 30 à $50 \mathrm{~cm}$, ou à assister les jeunes pousses naturelles. Ces plants issus de la régénération naturelle, ou élevés seulement pendant deux à quatre mois en pépinière, sont vulnérables à la dent des animaux et aux traumatismes divers qui les maintiennent vivaces sous forme de souches pendant plusieurs années (photo 1). Ces facteurs biophysiques entravent le développement des jeunes plants de baobab en milieu naturel (BATIONO, 2003 b). Ainsi le baobab est-il menacé dans presque tous les pays sahéliens où sa disparition est localement constatée (LYKKE, 1998).

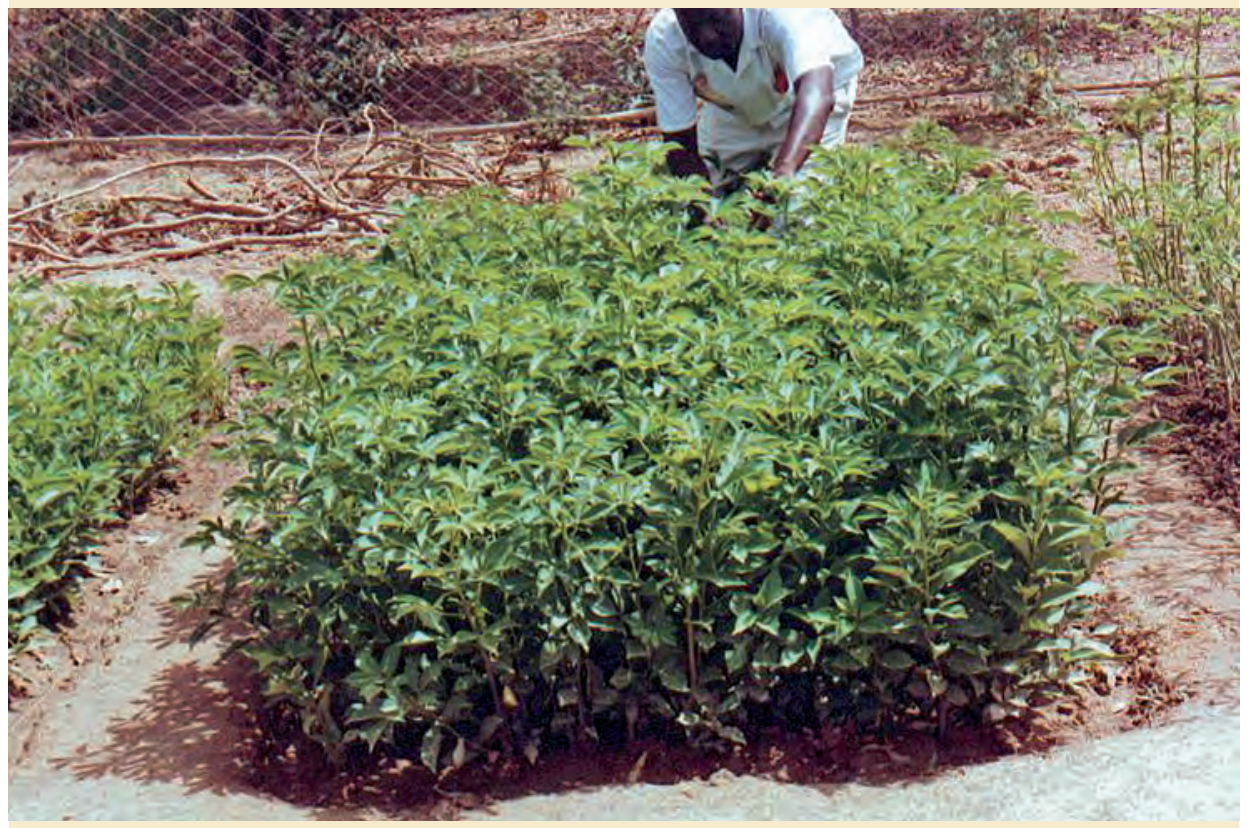

Photo 2.

Planche de baobab 40 jours après la levée.

Photo B. A. Bationo.

\section{Matériel et méthodes}

Les études conduites depuis 2000 par l'Icraf et les instituts de recherche agricole des pays sahéliens montrent que le baobab peut être cultivé comme des légumes dans les jardins (photo 2). Cette technique a été diffusée auprès des producteurs sahéliens, notamment au Mali et au Burkina Faso, et avait pour but de faciliter la récolte des feuilles et d'assurer la disponibilité de feuilles fraîches de baobab en saison sèche (BATIONO, 2003 a; SAVArd et al., 2006).

L'objectif de cette étude est de mieux appréhender la morphologie fonctionnelle des plants, la phénologie foliaire et la productivité des planches en feuilles fraîches en fonction des techniques de récolte des feuilles et de tester l'adaptabilité du baobab à la transplantation. L'apti tude à la transplantation permettrait d'envisager, dans une même planche, la production intensive de feuilles et de plants vigoureux de baobab pour régénérer rapidement les parcs agroforestiers par transplantation.
Les études sont conduites en station et en milieu paysan dans le plateau central du Burkina Faso. La pluviosité annuelle dans la zone est de l'ordre de 700 à $800 \mathrm{~mm}$. Les essais en station sont réalisés dans la pépinière de la station de recherche de Saria de l'Institut de l'environnement et de recherches agricoles (Inera), située au centreouest, à $80 \mathrm{~km}$ de Ouagadougou.

\section{Installation des planches de baobab}

Les planches de baobab utilisées ont une taille de $4,5 \mathrm{~m}^{2}$ ( $3 \mathrm{mx}$ $1,5 \mathrm{~m}$ ) chacune. Le sol à l'intérieur de chaque planche est remué jusqu'à $30 \mathrm{~cm}$ de profondeur pour permettre un bon développement des racines, avant d'y semer les graines d'Adansonia digitata traitées à l'acide sulfurique à $95 \%$ pendant $45 \mathrm{~min}$. L'objectif du traitement à l'acide est de lever la dormance tégumentaire des 


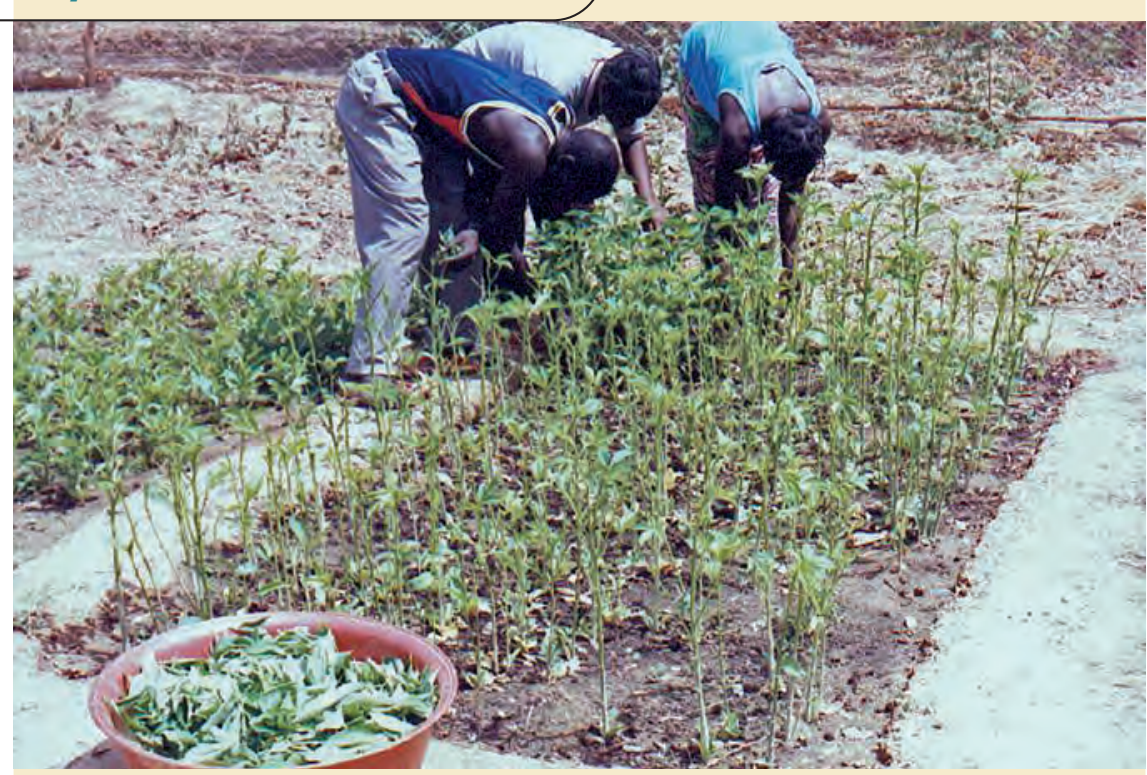

Photo 3.

Récolte des feuilles sur des pieds de jeunes baobabs de 40 jours en épargnant les bourgeons terminaux.

Photo B. A. Bationo.

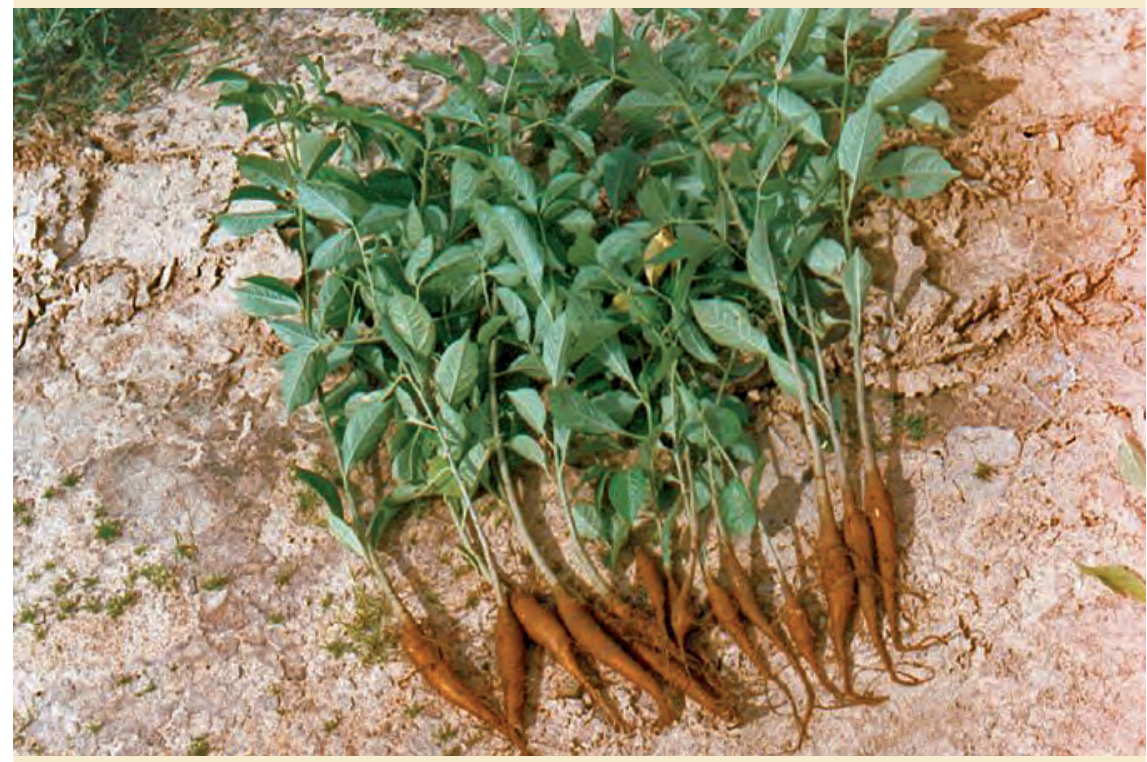

Photo 4.

Tubérisation des pivots racinaires de jeunes plants de baobab de deux mois (stade I) due à l'accumulation précoce de réserves.

Photo B. A. Bationo.

graines. La densité de semis est de $15 \mathrm{~cm} \times 15 \mathrm{~cm}$, soit environ 200 poquets par planche. Deux graines sont semées par poquet pour accroître les chances d'avoir au moins une levée par poquet. Le lot de graines utilisées a un taux de germination de $80 \%$. L'arrosage consiste à apporter quotidiennement durant la saison sèche deux arrosoirs d'eau, soit environ 20 litres au total par planche. Pendant l'hivernage, l'arro- sage est assuré par l'eau de pluie. Après la levée, les plantules sont démariées pour n'en maintenir qu'une par poquet. Les plantules obtenues du démariage servent à repiquer les éventuels poquets vides. Les planches sont sarclées une fois par mois pour éliminer la concurrence herbacée et amendées à raison de $2 \mathrm{~kg}$ de compost ou de fumier par planche et par mois.

\section{Techniques de récolte des feuilles}

Deux techniques de récolte des feuilles sont appliquées aux plants : la coupe rase à $5 \mathrm{~cm}$ au-dessus du sol (technique couramment utilisée par les producteurs) et la récolte sur pied en épargnant les bourgeons terminaux (photo 3). La dernière technique permet aux plants de poursuivre leur croissance en hauteur. Chaque technique de récolte est appliquée à trois planches. La périodicité des récoltes est mensuelle, sauf la première récolte qui a été faite 40 jours après la levée des semis. L'étude a été conduite sur une période de deux ans. Les données collectées portent sur la morphologie fonctionnelle des plantules, la vitesse de repousse des plants et la productivité en feuilles fraîches des planches en fonction de la technique de récolte.

\section{Transplantation des plants en milieu réel}

Trois stades de développement des plants soumis à la récolte sur pied ont été transplantés en saison pluvieuse en milieu naturel :

- stade I, plants âgés de 2 mois dont la hauteur est comprise entre $30 \mathrm{~cm}$ et $50 \mathrm{~cm}$;

- stade II, plants âgés d'un an dont la hauteur est comprise entre $1 \mathrm{~m}$ et $1,5 \mathrm{~m}$;

- stade III, plants âgés de deux ans dont la hauteur est comprise entre $2 \mathrm{~m}$ et $3 \mathrm{~m}$.

Pendant la plantation, les plants sont débarrassés de leurs feuilles sauf les 4-6 terminales et les bourgeons terminaux (traitement en rosette). Cinquante plants sont transplantés par stade de développement. La densité de plantation est de $10 \mathrm{~m}$ $\mathrm{x} 10 \mathrm{~m}$ et les plants mis en terre ne sont pas protégés contre les agressions extérieures. Les observations ont porté sur le taux de survie et la croissance en hauteur des plants un an après la plantation. 


\section{Résultats}

\section{Morphologie fonctionnelle des plantules}

Les plantules de baobab sont de type cryptogées, caractérisées par des cotylédons soudés à la base. Le système racinaire des plantules est initialement dominé par le pivot qui se tubérise progressivement pour atteindre 1,5 à $2,5 \mathrm{~cm}$ de diamètre au bout de deux mois (photo 4). Jusqu'à l'âge de deux ans, la profondeur d'enracinement demeure d'environ $50 \mathrm{~cm}$ et le système racinaire toujours dominé par le pivot (photo 5), ce qui rend moins pénibles les opérations de déterrement. En planche, certains plants développent des anastomoses entre leurs pivots racinaires (photo 6). La hauteur moyenne des plants en planche atteint 2,85 m (moyenne sur un effectif de 100 plants) deux ans après la levée.

\section{Productivité des planches et état des plantations}

L'intensité de la production des feuilles de baobab dans les planches est variable selon les saisons et la technique de récolte. Les périodes favorables à la production foliaire sont les saisons chaude et sèche ou chaude et humide, c'est-à-dire de mars à octobre. Les bourgeons sont en repos végétatif durant la période sèche et froide (novembre à mi-février) malgré l'arrosage. Durant cette période, les rejets après la coupe rase sont immédiatement suivis d'un arrêt de croissance. Pendant la période active, la productivité des planches soumises à la récolte sur pied est en moyenne de l'ordre de $0,4 \mathrm{~kg} / \mathrm{m}^{2} /$ mois de feuilles fraîches durant les deux premiers mois après la germination. Un an après la germination, la productivité moyenne est de l'ordre de $2 \mathrm{~kg} / \mathrm{m}^{2} /$ mois. Par contre, dans les planches où la coupe rase successive est pratiquée, les ébauches de rejets de souches n'apparaissaient qu'une à deux semaines après la coupe. La productivité moyenne un an après la germination est de $0,6 \mathrm{~kg} / \mathrm{m}^{2} /$ mois de feuilles fraîches pendant la période active (figure 1).

Le taux de survie des plants des stades II et III est de $100 \%$ et de $90 \%$ pour le stade I un an après la plantation. Le broutage par les animaux en divagation a cependant affecté la croissance des individus des stades I et II dont les hauteurs moyennes ont diminué respectivement de 35,22\% et $32,53 \%$ par rapport aux hauteurs moyennes au moment de la plantation (tableau I). En revanche, les animaux n'ont pas d'impact négatif significatif sur le développement des individus du stade III (photo 7) dont la hauteur moyenne des plants a augmenté de $5,76 \%$ et sur lesquels les producteurs ont commencé à récolter les feuilles une année après la plantation.

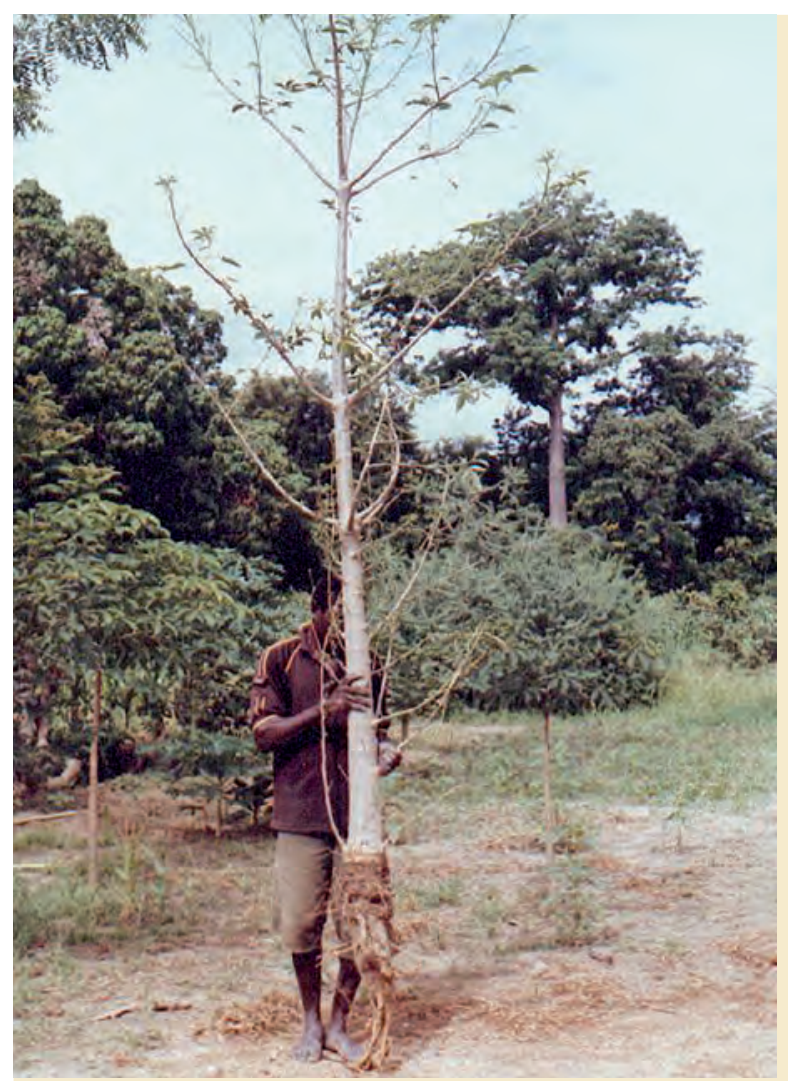

Photo 5.

Un pied de baobab élevé pendant deux ans (stade III) en planche.

Photo B. A. Bationo.

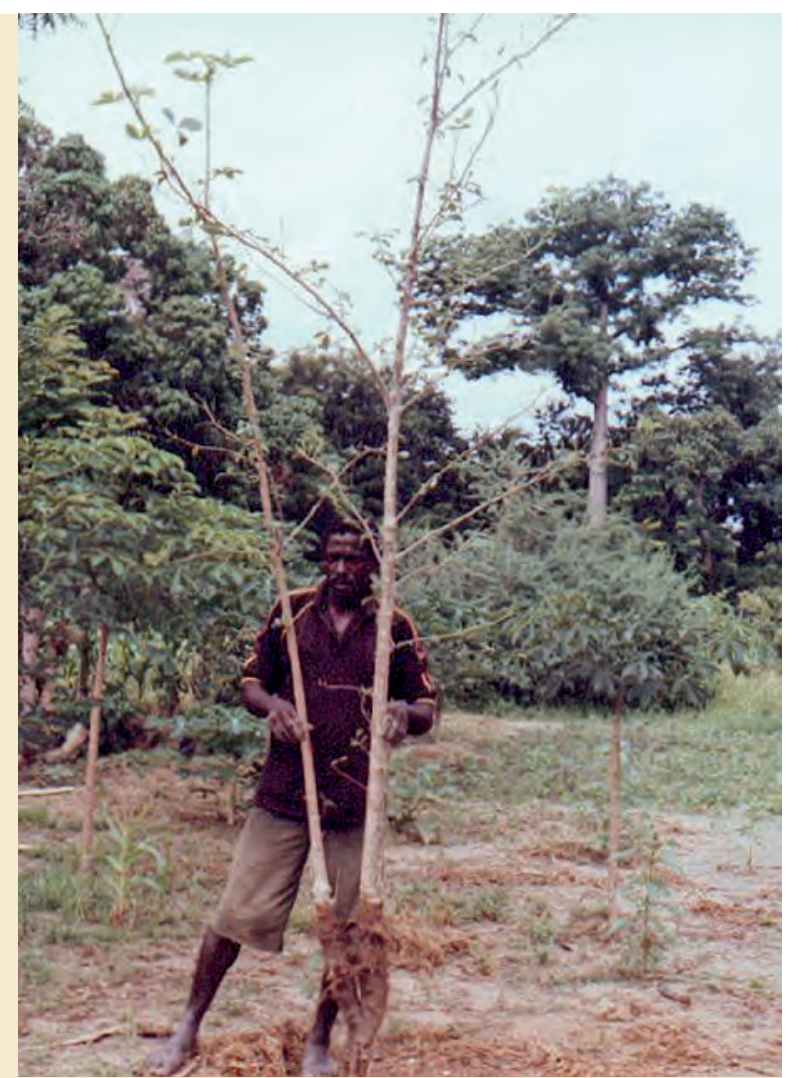

Photo 6.

Anastomoses entre les pivots racinaires de deux plants de baobab.

Photo B. A. Bationo. 


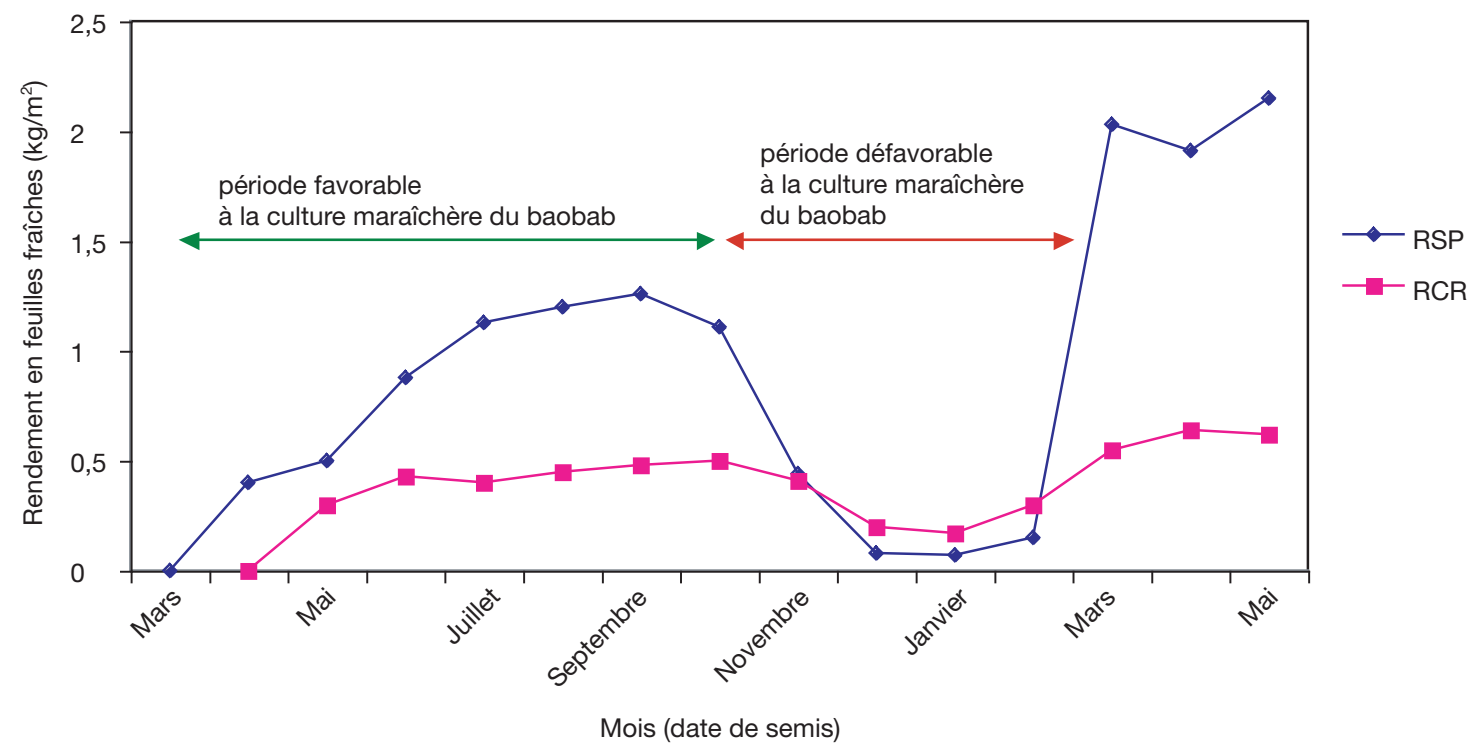

Figure 1.

Courbes de production de feuilles fraîches de baobab en planche en fonction de la technique de récolte pendant un cycle annuel. RSP : récolte sur pied en épargnant les bourgeons terminaux ; RCR : récolte par coupe rase.

\section{Discussion}

Le baobab est une espèce qui s'adapte aux conditions austères du Sahel (MAYDELL, 1983). Les plants survivent pendant plusieurs années aux traumatismes répétés, rejetant chaque année de souche. Ce statut est en partie lié à la morphologie fonctionnelle de ses plantules. La germination cryptogée, qui se caractérise par une forte activité des bourgeons cotylédonaires, assure une bonne capacité de rejet de souche des plants (JACKSON, 1974 ; MIQUEL, 1987). La tubérisation précoce du pivot racinaire est le résultat d'une accumulation de réserves (BATIONO et al., 2001) sur lesquelles la plantule vit pendant les périodes difficiles et durant les premiers moments après la transplantation. La technique de récolte foliaire sur pied, en épargnant les bourgeons terminaux, est la plus profitable. L'aptitude du baobab à la transplantation accroît davantage l'intérêt de cette technique de récolte. Elle permet de produire à la fois des feuilles et des plants à racines nues dans une même planche. Les plants sont entretenus en pépinière jusqu'à un stade de développement (stade III) où ils sont capables de résister aux traumatismes avant de les transplanter en milieu réel. L'aptitude à la transplantation permet ainsi de réduire le coût de production des plants en supprimant les dépenses liées à l'achat des pots plastique et les travaux liés à leur remplissage.
La phénologie foliaire des plants en planche indique cependant que la durée de la période (mars-octobre) de production optimale des feuilles n'est pas suffisamment différente de celle de la production des arbres adultes dans la nature (début mai-septembre). Dans les zones où les peuplements naturels sont abondants, la rentabilité financière directe des planches à baobab peut alors être limitée. En revanche, dans les zones où le baobab est quasi absent du paysage et où les populations s'approvisionnement principalement sur les marchés en toute saison, la culture maraîchère du baobab par les communautés rurales ou périurbaines permet d'accroître la disponibilité des feuilles et de produire des plants vigoureux pour enrichir les parcs agro-

Tableau I.

Taux de survie et croissance moyenne des plants de baobab un an après la plantation.

\begin{tabular}{|c|c|c|c|c|c|c|c|c|c|}
\hline & H1 (m) & $\begin{array}{l}\text { Stade I } \\
\text { H2 (m) }\end{array}$ & $\begin{array}{c}\text { Taux de } \\
\text { survie (\%) }\end{array}$ & $\mathrm{H} 1$ & $\begin{array}{c}\text { Stade II } \\
\text { H2 }\end{array}$ & $\begin{array}{c}\text { Taux de } \\
\text { survie (\%) }\end{array}$ & $\mathrm{H} 1$ & $\begin{array}{c}\text { Stade III } \\
\mathrm{H} 2\end{array}$ & $\begin{array}{c}\text { Taux de } \\
\text { survie (\%) }\end{array}$ \\
\hline$\Delta \mathrm{H}(\mathrm{m})$ & \multicolumn{2}{|c|}{$-0,14$} & & \multicolumn{2}{|c|}{$-0,41$} & & \multicolumn{2}{|c|}{0,15} & \\
\hline
\end{tabular}

$\mathrm{H} 1$ : hauteur moyenne des plants au moment de la plantation; $\mathrm{H} 2$ : hauteur moyenne des plants un an après la plantation ; $\Delta \mathrm{H}=\mathrm{H} 2-\mathrm{H} 1$. 
forestiers. Mais au-delà de la rentabilité financière directe des planches à baobab, c'est leur rôle dans le changement d'attitude des populations, la restauration des peuplements naturels et l'amélioration des conditions d'accès aux feuilles (photo 8) qui mérite d'être considéré. L'adoption du baobab en culture maraîchère peut contribuer à faire tomber au Sahel les mythes selon lesquels le baobab abrite des génies maléfiques qui causeraient la mort des personnes qui le plantent (BATIONO, 2003 b).

Pendant la période active, le débourrement continu des bourgeons et la ramification liée au développement des individus accroissent la productivité des planches, qui passe de $0,4 \mathrm{~kg} / \mathrm{m}^{2} /$ mois de feuilles fraîches durant les deux premiers mois après la germination à $2 \mathrm{~kg} / \mathrm{m}^{2} /$ mois de feuilles fraîches dans les planches âgées d'une année. La hauteur moyenne de l'ordre de $3 \mathrm{~m}$ atteinte par les plants en deux ans en planche invite à nuancer les propos de BHATNAGAR et al. (1993) selon lesquels les espèces ligneuses sahéliennes auraient une croissance lente. Le temps de latence d'une à deux semaines, nécessaire à l'apparition des ébauches de rejets sur les souches, augmente le délai entre deux récoltes consécutives par coupe rase. Cela ne permet pas de maintenir le rythme d'une récolte par mois. Cette technique, plus destructrice, ne favorise ni une productivité progressive en feuilles, ni la production de plants vigoureux capables de supporter les agressions en milieu naturel. L'arrêt précoce de la croissance des rejets après la récolte par coupe rase durant la période sèche et froide (période de repos végétatif) indique que les pousses durant cette période sont plus une réaction à la coupe qu'un comportement phénologique normal. Le développement d'anastomoses entre les racines de plants différents favorise des échanges physiologiques entre individus qui pourraient accroître la vitesse de propagation de maladies à l'intérieur de la planche de culture.

\section{Conclusion}

Les arguments développés en faveur de la culture du baobab en planche au Sahel sont généralement basés sur sa capacité à permettre la production de feuilles fraîches en saison sèche. La phénologie foliaire des plants en planche indique cependant qu'il ne faut pas exagérer cette capacité, parce que la période optimale de production foliaire, qui se situe de mars à octobre, n'est pas suffisamment différente de celle des arbres dans la nature au Sahel. Dans les zones où le peuplement naturel de baobab existe, les bénéfices de l'adoption des planches de baobab dans l'unique but de produire des feuilles peuvent être limités. L'aptitude à la transplantation du baobab permet d'élargir les objectifs des planches de baobab à la production à moindre coût de plants vigoureux à racines nues. La récolte des feuilles sur les jeunes plants, en épargnant les bourgeons terminaux, permet d'atteindre ce double objectif. Cette technique de récolte permet d'accroître la productivité des planches en feuilles et d'obtenir des plants vigoureux pour régénérer rapidement les parcs agroforestiers par transplantation. C'est pourquoi l'analyse des avantages des planches de baobab doit être à la fois économique, écologique et sociale en intégrant les possibilités de production de plants à moindre coût, la facilité d'accès aux feuilles et l'impact sur le changement de comportement des populations vis-à-vis des mythes liés à la régénération du baobab au Sahel.

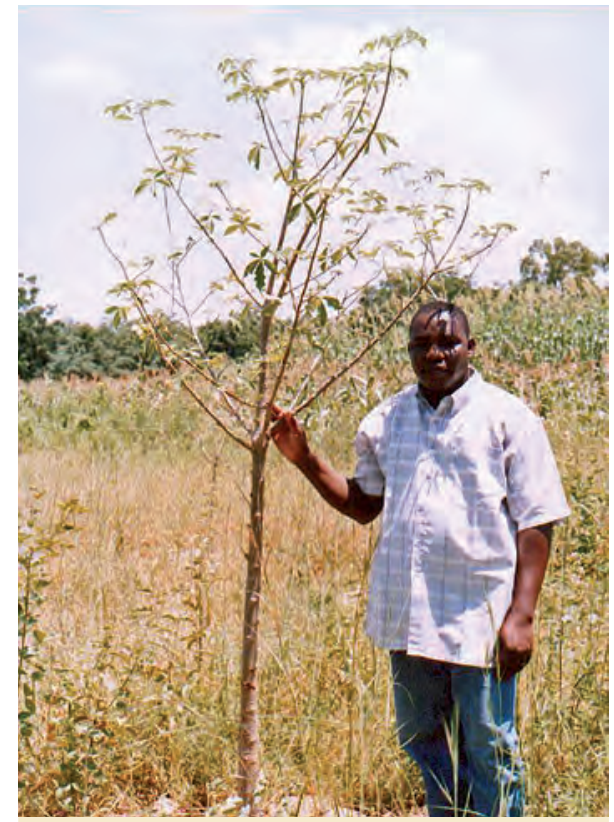

Photo 7.

Un pied de baobab au stade III un mois après sa transplantation en milieu naturel. Photo B. A. Bationo.

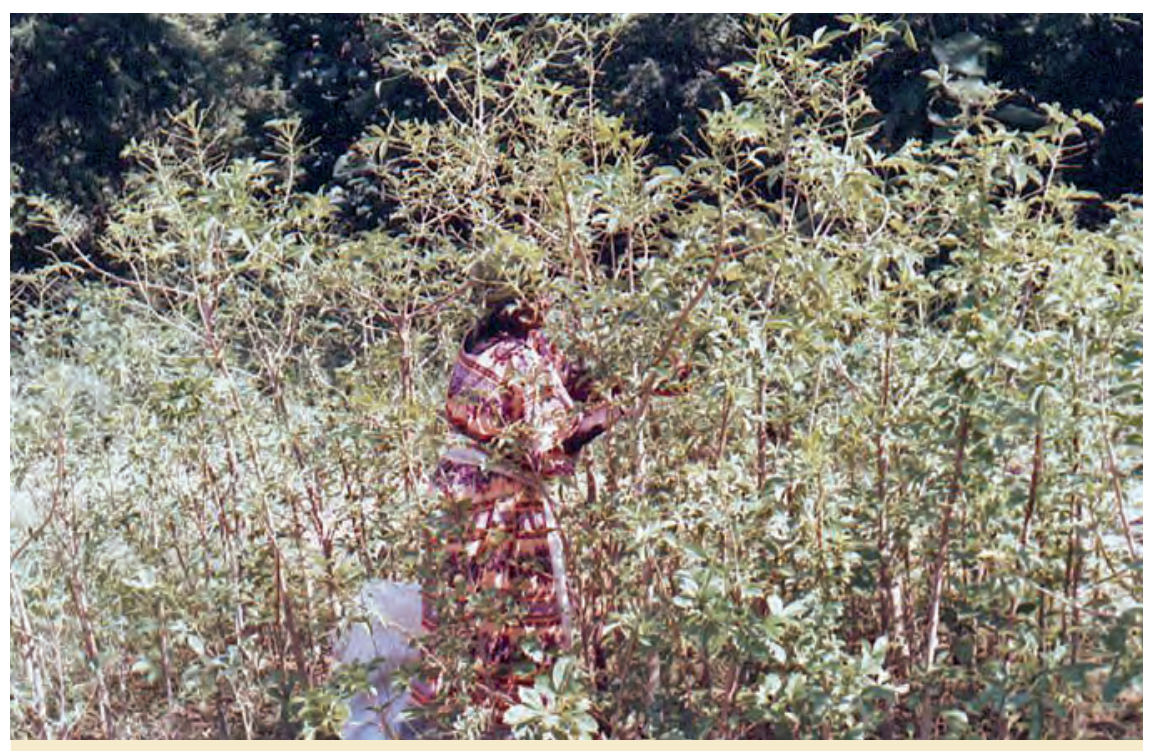

Photo 8.

Les planches de baobab facilitent l'accès et la récolte des feuilles fraîches de baobab. Ici une femme de plus de 60 ans qui s'adonne à la cueillette des feuilles. Photo B. A. Bationo. 


\section{Références bibliographiques}

BATIONO B. A., 2003 a. On peut cultiver le baobab comme la salade. Journal Évasion, n³72, p. 5.

BATIONO B. A., 2003 b. Étude de la structure et des contraintes socioculturelles à la régénération des parcs à baobab dans le Plateau Central du Burkina Faso. Ouagadougou, Burkina Faso, Inera/Icraf, 36 p.

BATIONO B. A., OUEDRAOGO S. J., GUINKO S., 2001. Stratégies de régénération naturelle de Detarium microcarpum Guill. et Perr. dans la forêt classée de Nazinon (Burkina Faso). Fruits, 56 : 271-285.

BHATNAGAR N., BHANDARI D. C., KAPOOR P., 1997. Competition in the early establishment phases of an even aged mixed plantation of Leucaena leucocephala and Acacia nilotica. Forest Ecology and Management, $57: 213-231$.

BONKOUNGOU E. G., AYUK E. T., ZOUNGRANA I., 1993. Les parcs forestiers des zones semi-arides d'Afrique de l'Ouest. Actes du Symposium international tenu à Ouagadougou, Burkina Faso, 25-27 octobre 1993. Nairobi, Kenya, Icraf, 226 p.

CES/AGF, 2000. Évaluation du taux de survie des plantations dans le plateau central du Burkina Faso. Yako, Burkina Faso, Ucp/Ces/Agf, 38 p.

CISSE M. I., 1995. Les parcs agroforestiers au Mali : état des connaissances et perspectives pour leur amélioration. Bamako, Mali, Icraf/Afrena, $53 \mathrm{p}$.

JACKSON G., 1974. Cryptogeal germination and other seedlings adaptation to the buring of vegetation in savanna region in the origin of pyrophytic habit. New Phytol, 73 : 771-780.
LAMIEN N., TRAORE S., 2002. Commercialisation des produits forestiers non ligneux dans la zone semi-aride du Burkina Faso : cas des feuilles sèches de baobab (Adansonia digitata L.). Bamako, Mali, Icraf, 12 p.

LEAKEY R. B., 1999. Farmers's toppriority fruit trees. Agroforestry Today, $11: 3-4$

LYKKE A. M., 1998. Connaissances et préférences locales concernant les plantes ligneuses du Sahel au Burkina Faso. In : Gestion des ressources naturelles au Sahel. Quelques exemples de l'initiative de recherche Serein, 1995-1997. Reenberg A., Nielsen I., Marsussen H. (éd.). Copenhague, Danemark, Serein Occasional Paper 7, p. 71-83.

MAYDELL H. J., 1983. Arbres et arbustes du Sahel : leurs caractéristiques et utilisations. Eschborn, Allemagne, Gtz, 631 p.

MIQUEL M., 1987. Morphologie fonctionnelle de plantules d'espèces forestières du Gabon, Bulletin du Muséum National d'Histoire Naturelle, 9 : 101-121.

NIKIEMA R., 1997. Commercialisation des produits alimentaires et forestiers provenant des parcs agroforestiers : cas des marchés de Zitentga et de Yako au Burkina Faso. In : Les parcs forestiers des zones semiarides d'Afrique de l'Ouest. Actes du Symposium international tenu à Ouagadougou, Burkina Faso, 25-27 octobre 1993. Bonkoungou E. G., Ayuk E. T., Zoungrana I. (éd.). Nairobi, Kenya, Icraf, p. 35-50.

OUEDRAOGO S. J., 1995. Les parcs agroforestiers au Burkina Faso. Bamako, Mali, Icraf, 76 p.

SAVARD V., OLIVIER A., FRANZEL S., 2006. Technique de production maraîchère de feuilles de baobab : potentiel d'adoption. Bois et Forêts des Tropiques, $287:$ 21-34. 